\title{
Multivariate Statistical Analysis of Time-of-Flight Secondary Ion Mass Spectrometry (ToF-SIMS) Spectral Images - Unbiased Analysis of the Entire 3-Dimensional Data Cube
}

\author{
Vincent S. Smentkowski \\ General Electric Global Research, 1 Research Circle, Niskayuna, NY, 12309
}

Time of flight secondary ion mass spectrometry (ToF-SIMS) has a number of desirable characteristics for surface analysis/microscopy including: high surface specificity (analysis of the top 0.3 to $1.0 \mathrm{~nm}$ of samples), high sensitivity ( $\mathrm{ppm}$ to $\mathrm{ppb}$ for all elements), high mass resolution $(\mathrm{M} / \Delta \mathrm{M}>10,000)$, high lateral resolution $(<100 \mathrm{~nm})$, and the ability to detect all elements plus molecular fragments during a single measurement [1]. At each pixel in a ToFSIMS image an entire mass spectrum is saved in the raw data file generating a three-dimensional data cube that is often referred to as a spectral image. The spectral image data files contain approximately $8 \times 10^{10}$ spectral channels, which translate to a wealth of information regarding the measurement. Few tools are available to assist the analyst in visualizing the entire raw data set and as a result, most of the data are not utilized. The ToF-SIMS analyst typically relies on information known about the material, results obtained from similar past analysis, high peak intensities, and/or unusual species detected in the ToF-SIMS spectrum to manually select a few species for further analysis. The ToF-SIMS analyst is expected to perform rapid, cost effective analysis, and provide a complete evaluation of the dataset. Figure 1 shows a typical ToF-SIMS spectrum collected on a polymer sample. Note that, unlike an EDS spectrum from an SEM for example, there are significantly more peaks corresponding to the elements, molecules, and fragments detected.

Automated, non-biased, multivariate statistical analysis (MVSA) techniques are useful for converting the massive amount of data into a smaller number of chemical components (spectra and images) that are needed to fully describe the ToF-SIMS measurement [2-3]. At GEGR, we are using the AXSIA (Automated eXpert Spectrum Image Analysis) toolkit developed at Sandia National Laboratories [4] to perform MVSA on ToF-SIMS spectral images. The advantages of AXSIA include: the ability to select the mass range, the ability to bin the mass spectra from $0.001 \mathrm{amu}$ to $1.0 \mathrm{amu}$, optimally scaling the data to account for Poisson counting statistics [5], and intuitive results (e.g., negative peaks are not allowed in the spectral response). The AXSIA analyses were performed on a Dell Latitude D400 laptop computer with a $1700 \mathrm{MHz}$ processor and 1.046 GB RAM using Microsoft Windows 2000. All of the AXSIA analyses that will be presented required less than 30 seconds.

AXSIA analysis of the ToF-SIMS spectrum shown in Figure 1 provided 5 components. The component spectra and images generated by AXSIA are shown in Figure 2. The AXSIA analysis was performed using the entire mass spectrum ( 0 to 1,200 amu); for clarity, only the low mass ( 0 to $150 \mathrm{amu}$ ) regions of the spectra are shown in Figure 2. In order to decrease the file size (increase computation speed) this data set was binned to $1 \mathrm{amu}$. The five components fully describe the data. Component 1 is characteristic of the polymer, component 2 is rich in alkali species, component 3 represents polymer additive (Bn POSS) rich region of the sample, component 4 is characteristic of silicone oils (sample contaminant) and component 5 is characteristic of hydrocarbon species (contaminants) on the sample. 
In the presentation, numerous additional examples will be discussed, stressing the analytical insight provided by AXSIA analysis.

References:

[1] J. C. Vickerman, and D. Briggs, D., ToF-SIMS Surface Analysis by Mass Spectrometry, Surface Spectra/IMPublications: UK, 2001.

[2] J. A. Ohlhausen, M.R. Keenan, P.G. Kotula, P.G., and D.E. Peebles, Appl. Surf. Sci., 231-232 (2004) 230.

[3] V.S. Smentkowski, J.A. Ohlhausen, P.G. Kotula, and M.R. Keenan, Appl. Surf. Sci., 231-232 (2004) 245.

[4] M.R. Keenan, and P.G. Kotula, U.S. Patent No. 6,584,413, June 24, 2003; M.R. Keenan, P.G. Kotula, U.S. Patent No. 6,675,106, Jan 6, 2004.

[5] M.R. Keenan, and P.G. Kotula, Surf. Interface Anal., 36 ( 2004) 203.
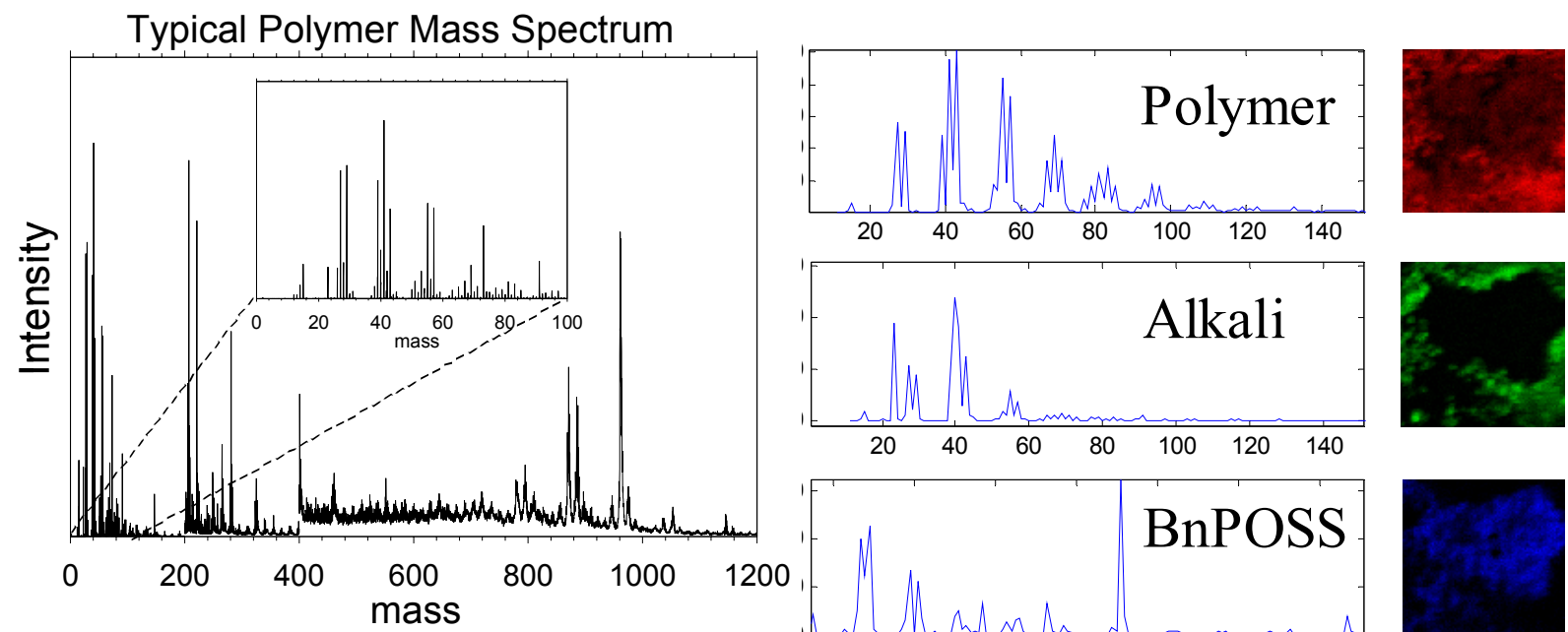

Fig. 1. ToF-SIMS spectrum of a typical polymer.
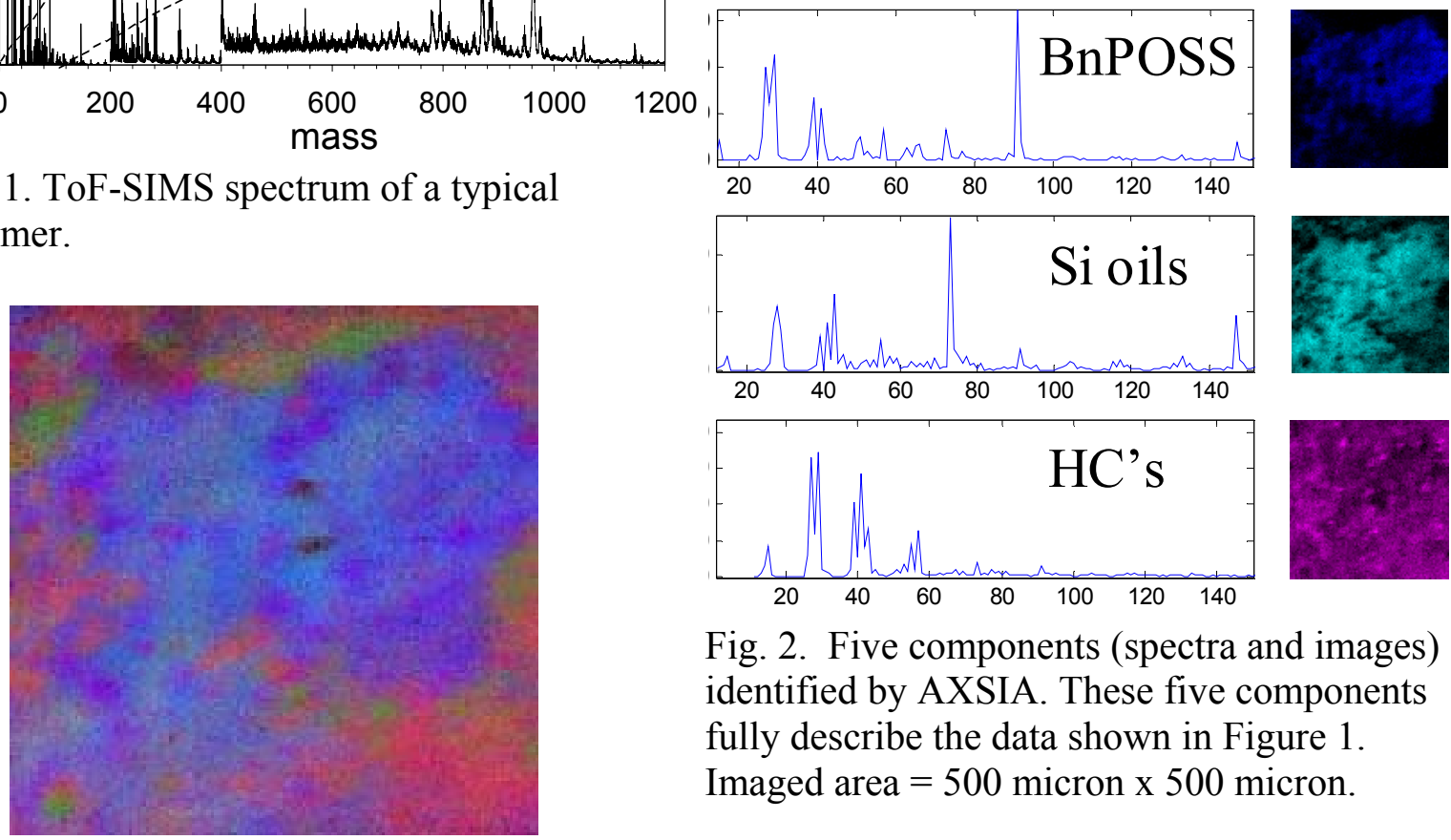

Fig. 2. Five components (spectra and images) identified by AXSIA. These five components fully describe the data shown in Figure 1. Imaged area $=500$ micron $\times 500$ micron.

Fig. 3. Color overlay of the AXSIA analysis (imaged area is 500 micron x 500 micron). 